\title{
Comparison of reversible and irreversible dipolar assemblies in a ferrofluid
}

\author{
M. Klokkenburg*, B.H. Erné \\ Van't Hoff Laboratory for Physical and Colloid Chemistry, Debye Institute, Utrecht University, Padualaan 8, 3584 CH Utrecht, The Netherlands
}

Received 19 October 2005

Available online 29 March 2006

\begin{abstract}
Zero-field aggregation of magnetic nanoparticles in a ferrofluid can either be irreversible or result from a dynamic equilibrium; the two cases can be distinguished by measurements of the complex magnetic susceptibility and by cryogenic transmission electron microscopy (cryo-TEM). We demonstrate this by comparing two colloidal systems that show dipolar structure formation in zero field. A dispersion of magnetic iron nanoparticles is gradually oxidized to decrease the magnetic moments, and despite the vanishing dipolar attractions, thermal motion does not break up the dipolar structures into single particles. Instead, the dipolar structures become chemically fixed during the oxidation process, an example of irreversible aggregation. In contrast, the zero-field dipolar structures in a chemically stable magnetite dispersion are found to disintegrate upon dilution, indicating that the structures are reversible and result from a dynamic equilibrium.
\end{abstract}

(C) 2006 Elsevier B.V. All rights reserved.

PACS: 75.50.M; 75.30.C; 36.40.C

Keywords: Ferrofluids; Dipolar structures; Iron; Oxidation; Magnetite; Dynamic magnetic susceptibility; Cryogenic transmission electron microscopy

\section{Introduction}

Ferrofluids are colloidal dispersions of single-domain magnetic nanoparticles, electrostatically or sterically stabilized in water or organic solvents. When the magnetic interactions between the particles are sufficiently strong compared to thermal energy, theory and simulations predict anisotropic aggregation in zero field [1,2]. In principle, the size of the structures should be determined by a dynamic equilibrium between growth of the structures by addition of particles and shrinking of the structures by removal of particles. Two methods can be used to test the reversibility of dipolar chain formation: to decrease the dipole moment of the particles or to decrease the concentration. Both should shift the balance from enthalpy-driven chain growth to entropy-driven disintegration into smaller units.

\footnotetext{
*Corresponding author. Tel.: +3130 2532888; fax: + 31302533870 .

E-mail address: m.klokkenburg@chem.uu.nl (M. Klokkenburg).
}

Here, we use this knowledge to examine the difference in behavior between two systems, an oxidized iron system with irreversible aggregates and a magnetite system with reversible dipolar chain formation $[3,4]$. In the first case, we gradually oxidize surfactant-stabilized iron metal particles dispersed in an organic solvent. The magnetic moments are decreased by the oxidation of iron to iron oxides [6-10], which have a considerably lower saturation magnetization. Since anisotropic aggregation is due to magnetic interactions, weakening the magnetic moments is expected to break up the dipolar structures. In the second case, we systematically decrease the particle concentration of a dispersion of chemically stable magnetite particles. Upon dilution, dynamic assemblies should break up for entropy reasons. The effects on the size of the structures are ascertained using two techniques: complex magnetic susceptibility measurements [5] and cryogenic transmission electron microscopy (cryo-TEM) [3,4]. The first technique informs about the macroscopically averaged behavior, and the second technique provides images of the structures with single-particle resolution. 


\section{Experimental section}

The initial iron dispersions were prepared and characterized by Butter et al. $[3,9,11]$. These ferrofluids with codes $\mathrm{A}, \mathrm{B}$, and $\mathrm{C}$ contain unoxidized iron-carbon particles in Decalin with the stoichiometry of $\mathrm{Fe}_{0.75} \mathrm{C}_{0.25}$ (saturation magnetization: $1.49 \times 10^{6} \mathrm{~A} \mathrm{~m}^{-1}$ ) and have the radii listed in Table 1 . The particles are coated with an organic surface layer (polyisobutene) [12] of 6 to $7 \mathrm{~nm}$. This relatively thick polymer layer is necessary to prevent the metal particles from clustering and effectively reduces van der Waals interactions between the particles, to ensure that dipolar interactions are dominating [3,9,11]. The colloidal magnetite dispersion (D) was prepared by a method previously described [4], in which the particles are coated with a thin surfactant layer of $2 \mathrm{~nm}$ consisting of oleic acid and oleyl amine (Table 1).

The procedure used to oxidize the iron particles of ferrofluids A, B, and C is described in detail in Section 3.1.1. Initial and final magnetic radii of the particles calculated from magnetization curves are listed in Table 1 assuming the presence of single particles (a correct assumption for ferrofluid $\mathrm{A}$ and a fair approximation for ferrofluid $\mathrm{B}$; the slow dynamics of ferrofluid $\mathrm{C}$ made its magnetization curves difficult to interpret). The curves were recorded on a Micromag 2900 alternating gradient magnetometer from Princeton Measurements Corporation. The ferrofluids that contained partially oxidized iron particles were stable for months.

Magnetic susceptibility measurements at $875 \mathrm{~Hz}$ were performed with a Kappabridge KLY-3 susceptibility meter from Agico. Dynamic susceptibility spectra in the $3-10^{5} \mathrm{~Hz}$ range were recorded using a home-built setup [5] with mutually inducing coils (Helmholtz configuration). All measurements were done at room temperature. The interaction energy of the magnetic field $\left(30 \mathrm{~A} \mathrm{~m}^{-1}\right.$ amplitude) with the iron(oxide) particles was on the order of $10^{-2} k_{\mathrm{B}} T$. As a result of the weak field it was assumed that the forces acting on the particles did not influence the systems.

Cryo-TEM images were made by preparing a vitrified film of dispersion (mass fraction iron: 0.002; mass fraction iron oxide: 0.03 ) in a nitrogen atmosphere on grids with holey carbon film (R2/2 Quantifoil Micro Tools, Jena, Germany). The samples were prepared by a vitrobot [13], thereby removing excess solution by blotting (iron: $1 \times 3 \mathrm{~s}$ blotting; iron oxide: $1 \times 2 \mathrm{~s}$ blotting). CCD-images were taken on a Philips Tecnai 12 TEM operating at $120 \mathrm{kV}$ using low-dose techniques.

\section{Results and discussion}

\subsection{Arrested structures: oxidized metallic iron ferrofluids}

\subsubsection{Oxidation procedure}

To oxidize the metallic iron particles, typically $3 \mathrm{~mL}$ of dispersion was introduced in a narrow nitrogen-filled Schlenk flask, which was subsequently shortly opened to air. The oxidation kinetics of the iron particles are illustrated in Fig. 1a. As depicted in Fig. 1a for ferrofluid $\mathrm{A}$, the magnetic susceptibility at $875 \mathrm{~Hz}$ decreases exponentially over several days, after the ferrofluid in the immobile flask has been shortly exposed to air. After $24 \mathrm{~h}$, oxidation is slowed down and a passivating iron oxide layer has formed [11]. Changing the duration of aeration from 1.5 to $5 \mathrm{~min}$ only weakly influences the curve, which suggests that the solvent rapidly saturates with oxygen upon exposure to air. For better control of the oxidation, a stepwise treatment was adopted. The gas above the ferrofluid was replaced by air within $5 \mathrm{~s}$, and the Schlenk flask was closed again. The air now had little time to enter the liquid while the flask was open, and therefore the amount of oxygen admitted into the system corresponded to about $17 \mathrm{~mL}$ of air. After adding each dosage of air, the ferrofluids were stirred for $24 \mathrm{~h}$ or more, in line with the reaction kinetics in Fig. 1a. The result was that the magnetic susceptibility at $3 \mathrm{~Hz}$ now decreased more or less to the same extent in each oxidation step (Fig. 1b). This suggests full reaction of the oxygen admitted to the Schlenk flask in each oxidation step. For all the ferrofluids, the data now indicate the gradual oxidation of the magnetic particles and give no evidence of passivation due to the iron oxide layer.

\subsubsection{Effect of oxidation on dipolar structure formation}

Fig. 2 shows the dynamic susceptibility spectra of ferrofluids $\mathrm{A}-\mathrm{C}$ as a function of the number of oxidation steps. Ferrofluids A-C correspond to different situations and are now discussed separately. In A, rotational motion of single particles dominates, while in $\mathrm{B}$ and $\mathrm{C}$ it is thermal motion of short or long dipolar chains.

Table 1

Core radii and concentrations of the iron ferrofluids (A-C) [11] and the magnetite ferrofluid (D) [4]

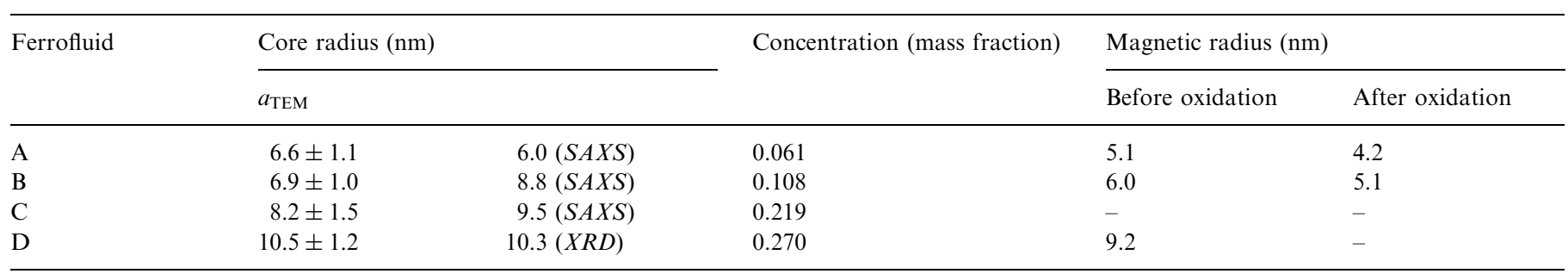



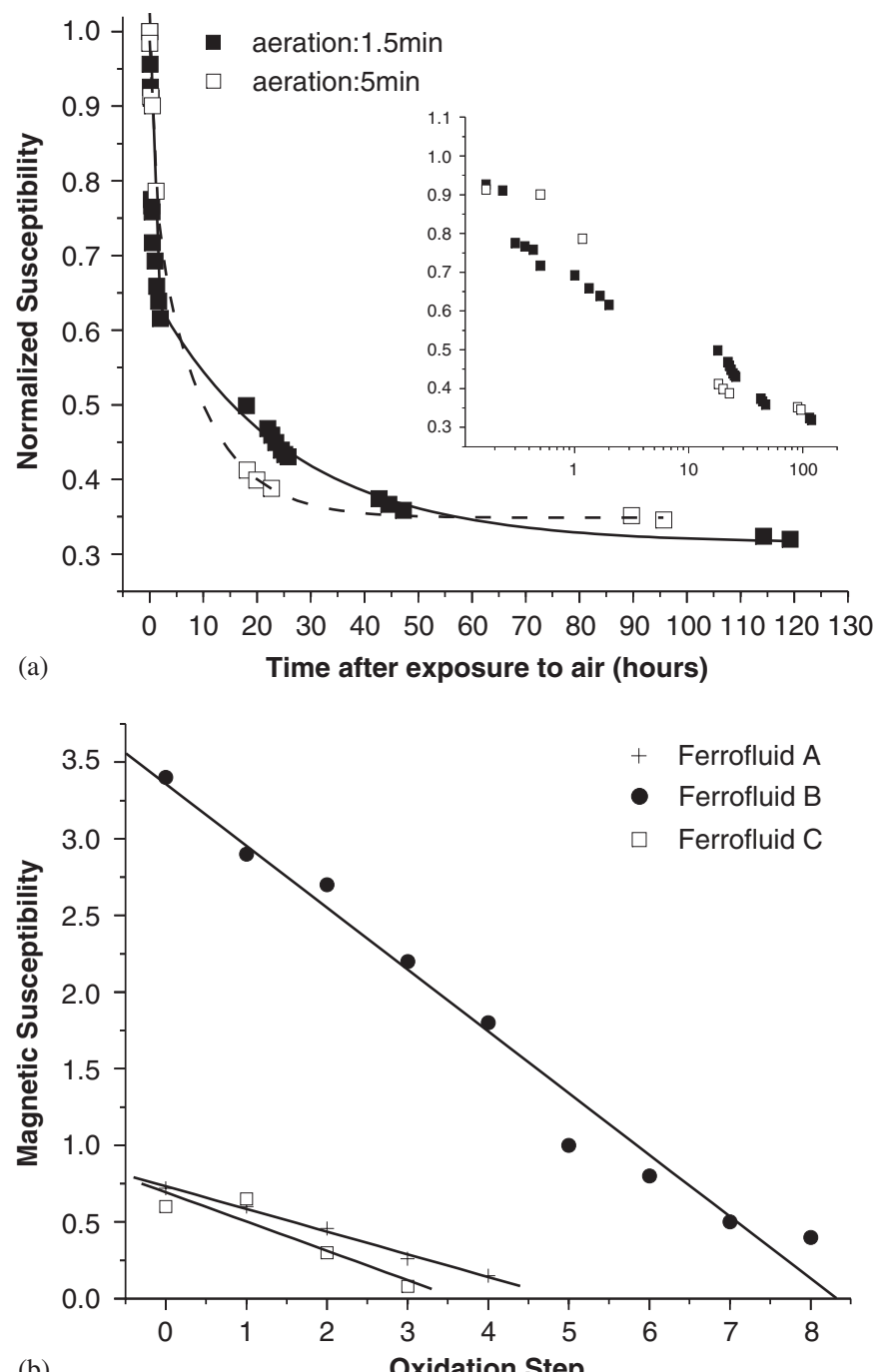

(b)

Oxidation Step

Fig. 1. (a) Normalized initial magnetic susceptibility of ferrofluid A at $875 \mathrm{~Hz}$, as a function of time after aeration for $1.5 \mathrm{~min}$ or $5 \mathrm{~min}$. The inset shows the normalized susceptibility on a logarithmic time scale. (b) Absolute magnetic susceptibility at $3 \mathrm{~Hz}$ after consecutive oxidation steps ( $5 \mathrm{~s}$ of aeration, $24 \mathrm{~h}$ or more of stirring) for ferrofluids A-C.

3.1.2.1. Ferrofluid A. In ferrofluid A, no dipolar chains are present [5], and consequently, there should also only be single particles after oxidation, except if chemical instability causes aggregation. At low frequencies the initial magnetic susceptibility is given by

$\chi_{i}=\frac{\mu_{0} \mu^{2} N}{3 k_{\mathrm{B}} T V}$,

where $\mu_{0}$ is the permeability of vacuum, $\mu$ is the magnetic dipole moment, $\mu N / V=M_{\mathrm{s}}$ is the saturation magnetization of the ferrofluid, with a number $N$ of colloids per volume $V, T$ is the absolute temperature and $k_{\mathrm{B}}$ is the Boltzmann constant. The dipole moment of the particles is given by

$\mu=(4 / 3) \pi a_{\mathrm{M}}^{3} m_{\mathrm{s}}$,

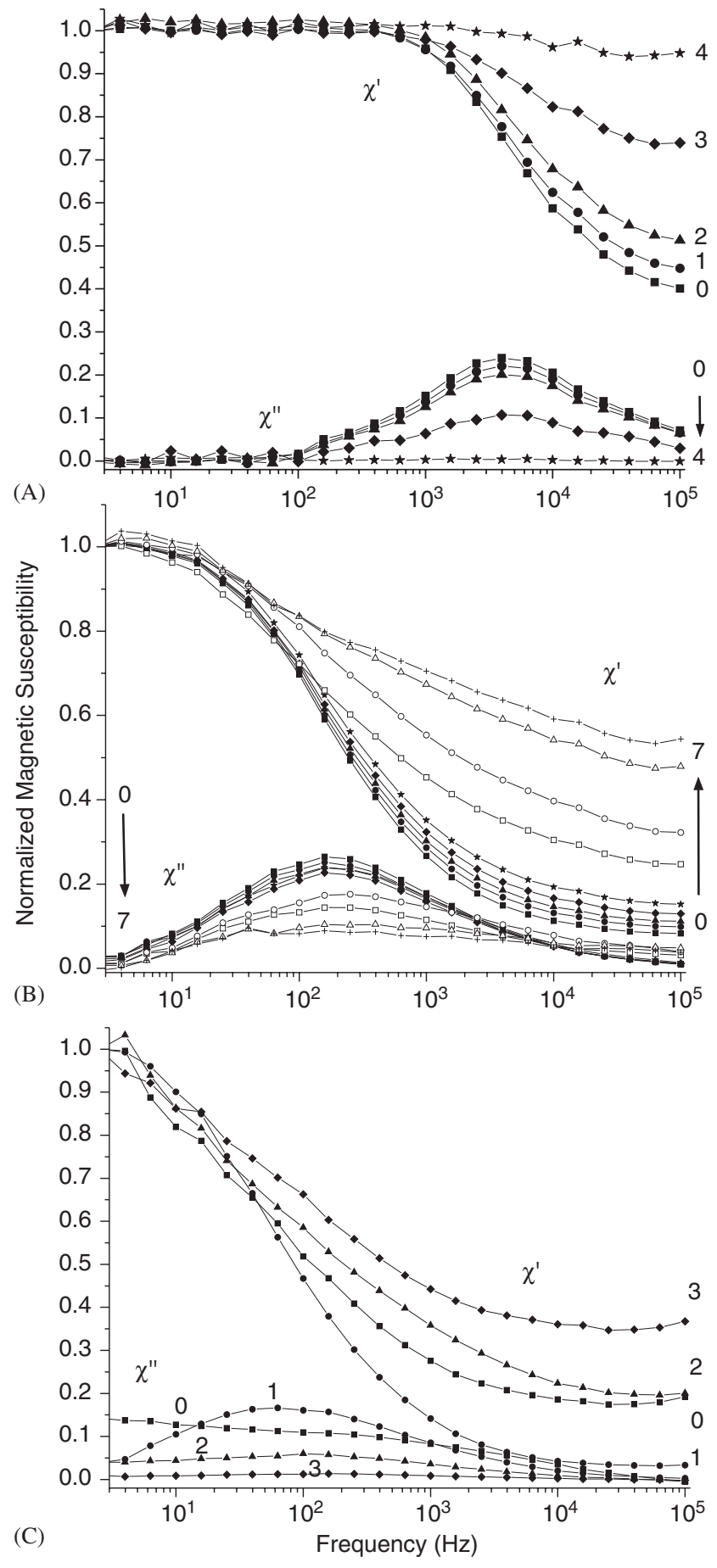

Fig. 2. Frequency dependence of the real $\left(\chi^{\prime}\right)$ and imaginary $\left(\chi^{\prime \prime}\right)$ components of the normalized magnetic susceptibility of metallic iron ferrofluids A-C upon oxidation. The spectra are scaled by normalizing the real value at $3 \mathrm{~Hz}$. The numbers close to the curves indicate the different oxidation steps (see Fig. 1b).

where $a_{\mathrm{M}}$ is the magnetic radius and $m_{\mathrm{s}}$ is the saturation magnetization of the magnetic material.

For unoxidized ferrofluid A, the particles have a characteristic frequency $\left(\omega_{\text {char }} / 2 \pi\right)$ of $4 \mathrm{kHz}$. This can be compared to a value of $8 \mathrm{kHz}$ expected for Brownian 
rotation of non-interacting spherical particles [5],

$\omega_{\mathrm{B}}=\frac{k_{\mathrm{B}} T}{4 \pi \eta a_{\mathrm{h}}^{3}}$,

where $T$ is the temperature, $\eta$ is the viscosity of the solvent $\left(2.5 \times 10^{-3} \mathrm{~kg} \mathrm{~m}^{-1} \mathrm{~s}^{-1}\right)$, and $a_{\mathrm{h}}$ is the hydrodynamic radius $\left(a_{\mathrm{h}}=a_{\text {core }}+7 \mathrm{~nm}\right)$. The polymer shell thickness of 6-7 nm is an effective thickness that is based on model-dependent fitting of SAXS data [9] and assumes that the shell has a homogeneous density. In reality, the shell probably has a density that is relatively homogeneous near the surface of the iron particle but decreases near the edge of the shell. The polymer shell therefore extends to a few nanometers more than $6-7 \mathrm{~nm}$, so that a larger value should be used in the calculation of the hydrodynamic radius. Cryo-TEM clearly demonstrates that the particles do not form any aggregates [3]. We conclude that the characteristic frequency of $4 \mathrm{kHz}$ is the empirically found value for single sphere rotation in our system.

As a result of polydispersity, relatively small particles are also present, with characteristic frequencies above $100 \mathrm{kHz}$, associated with rotation of magnetic moments inside the particles. The characteristic frequency of this so-called Néel relaxation is given by

$\omega_{\mathrm{N}}=2 \pi f_{0} \exp \left(\frac{-K V_{\mathrm{M}}}{k_{\mathrm{B}} T}\right)$,

where $f_{0}$ is a constant in the range $10^{7}-10^{12} \mathrm{~Hz}[14,15], K$ is a shape-dependent material constant, and $V_{\mathrm{M}}$ is the magnetic domain volume, that is $V_{\mathrm{M}}=\left(\frac{4}{3}\right) \pi a_{\mathrm{M}}^{3}$ for a spherical domain. Comparing Eq. (3) with Eq. (4) shows that Néel rotation strongly depends on the magnetic radius of the particle, whereas Brownian rotation is more weakly dependent on the hydrodynamic radius. The fastest rotation mechanism dominates for individual particles [14].

The characteristic frequency of $4 \mathrm{kHz}$ for particles that relax by Brownian rotation hardly changes upon oxidation, despite the decline of the dipole moment. The explanation for this observation is twofold. First, the hydrodynamic radius is only weakly affected when the particles are oxidized. The decrease in magnetic susceptibility by a factor 3 observed for system A (Fig. 1b), requires only the oxidation of a $1 \mathrm{~nm}$ shell of metal, yielding a $1.5 \mathrm{~nm}$ shell of oxide. For a particle with a radius of $13.6 \mathrm{~nm}, 0.5 \mathrm{~nm}$ growth corresponds to less than $5 \%$, so that the characteristic frequency for Brownian rotation only shifts by 0.06 on a logarithmic scale, imperceptible in Fig. 2 where the data points are 0.2 apart. Second, relaxation that is initially dominated by Brownian rotation is taken over by Néel rotation after partial oxidation. Consequently, since Néel relaxation occurs at much higher frequencies, the particles do not contribute anymore to the signal at $4 \mathrm{kHz}$, leaving the Brownian rotation rate almost unchanged.

After four oxidation steps, relaxation via rotation of the whole particle is completely taken over by rotation of the magnetic moment inside the particles, and an almost flat spectrum is reached. The characteristic frequency is then beyond our experimental range. The constant characteristic frequency of the particles that do continue to relax by Brownian rotation agrees with the lack of dipolar structures before or after oxidation.

3.1.2.2. Ferrofluid B. Before oxidation, ferrofluid B consists of dynamic dipolar structures with an average of about four particles per chain [3,5]. The characteristic frequency is strongly affected by the number of particles in a dipolar chain [16] and, therefore, $\omega_{\text {char }} / 2 \pi$ is now situated at $200 \mathrm{~Hz}$ instead of the order of $4 \mathrm{kHz}$ for single particles.

The iron particles were oxidized in eight consecutive steps, leading to a progressive decrease of the susceptibility. The decrease of the low-frequency limit of the susceptibility to $15 \%$ of its initial value corresponds to a similar drop of the magnetic coupling parameter $\lambda$ since both scale with the dipole moment $\mu$ squared (compare with Eq. (1)):

$\lambda=\frac{\mu_{0} \mu^{2}}{4 \pi k_{\mathrm{B}} T \sigma^{3}}$,

where $\sigma$ is the hard sphere diameter. Dipolar structure formation is expected for $\lambda>2[1,2]$. For unoxidized ferrofluid B, $\lambda$ equals 19 , in line with the observation of dipolar structures in the unoxidized ferrofluid (Fig. 3a). However, after eight oxidation steps the interaction parameter is estimated at 2.9 (15\% of 19). Therefore, the equilibrium cluster-size is also expected to decrease considerably.

The theoretical prediction is not experimentally confirmed by the dynamic susceptibility measurements. Both the shape and the characteristic frequency remain the same after each oxidation step (Fig. 2). If the chains would break upon oxidation, the characteristic frequency would gradually shift from $200 \mathrm{~Hz}$, the value for the initial chains, to about $4 \mathrm{kHz}$, the value for single particles (compare with the results in Section 3.2). Instead, even though the magnetic moments decrease, Brownian relaxation at $200 \mathrm{~Hz}$ is directly replaced by Néel relaxation above $100 \mathrm{kHz}$. The chains are oxidized without breaking up.

Cryo-TEM images of ferrofluid B support the continued presence of chain-like structures in the fluid after oxidation, as shown in Fig. 3. The average chain length does not appear to have changed compared to the situation before oxidation [3]. Fig. 3b reveals an iron oxide layer of about $1.2 \mathrm{~nm}$ around the unoxidized iron cores and indicates that most of the oxide layers touch each other. The dipolar structures apparently become chemically fixed when the ferrofluid is exposed to air.

3.1.2.3. Ferrofluid C. In ferrofluid C, most of the particles are in aggregates that are much larger than in ferrofluid B (Fig. 4a), and therefore a broad distribution around very low frequencies is observed in the dynamic magnetic susceptibility spectra (Fig. 2C). Contrary to the spectra of ferrofluid $\mathrm{B}$, the low-frequency limit is below $3 \mathrm{~Hz}$. In spectrum 1 of ferrofluid $\mathrm{C}$, after the first oxidation 


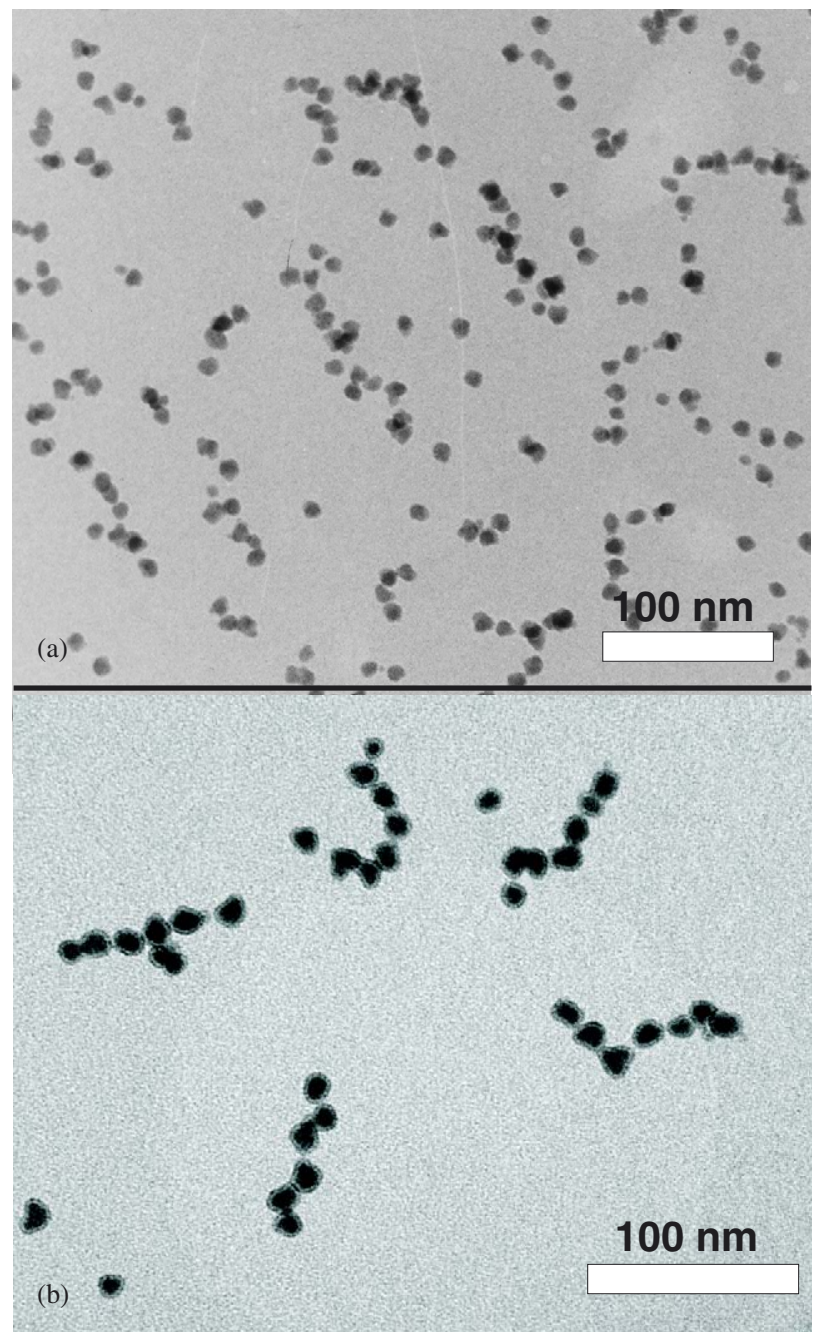

Fig. 3. Typical in situ cryo-TEM images in zero field of vitrified dispersions of ferrofluid B, containing (a) unoxidized [3,11] and (b) oxidized particles with a magnetic radius of $5.1 \mathrm{~nm}$ (b). The oxidized particles still show the chains formed before oxidation. Oxide shells of $1.2 \mathrm{~nm}$ are visible due to the lower contrast of iron oxides compared to metallic iron.

step, a peak in the imaginary part becomes visible at $65 \mathrm{~Hz}$, which suggests that the large structures in the unoxidized ferrofluid disintegrate into smaller units, rotating at higher frequencies.

In the next oxidation steps, the peak at $65 \mathrm{~Hz}$ disappears, the number of Néel-relaxing particles increases, and little change is seen in the shape of the spectrum, suggesting that the structures have become fixed, as observed for ferrofluid $\mathrm{B}$, and that no additional single particles have been obtained. These conclusions are confirmed by cryo-TEM images of oxidized ferrofluid C (Fig. 4b), which indicate the presence of aggregates that are smaller than before oxidation (Fig. 4a).

The appearance of a peak at $65 \mathrm{~Hz}$ is not consistent with a shift in a dynamic equilibrium between chains of different sizes. Such an equilibrium would lead to a continuous size distribution of the aggregates which gradually shifts to

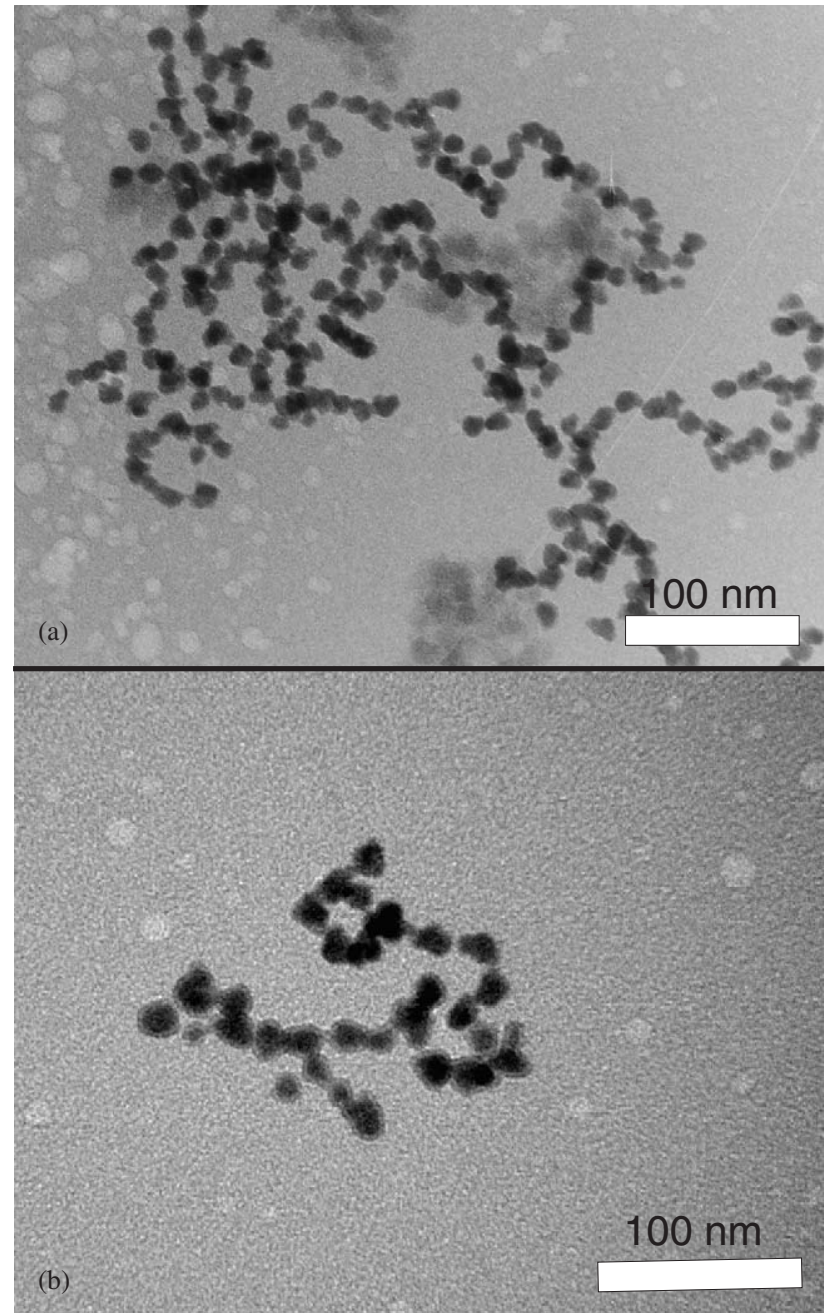

Fig. 4. Typical in situ cryo-TEM images in zero field of vitrified dispersions of ferrofluid $\mathrm{C}$, containing (a) unoxidized [3,11] and (b) partially oxidized particles.

higher frequencies upon dilution (see Section 3.2), not mainly to the appearance of units that respond at about $65 \mathrm{~Hz}$. Our tentative explanation is that chemical fixation of the large network structures is not perfectly homogeneous but that a distribution exists in the strength of the oxidic links between the particles. At a certain point of the oxidation process, subunits of the networks are already frozen, but the subunits are separated by sites where the link between two particles is relatively weak. Thermal motion causes these relatively stable units to break off from the large structures.

An interesting detail can be observed in the evolution of the normalized real component $\chi^{\prime}$ at $100 \mathrm{kHz}$ : upon oxidation, it first decreases before later increasing. On an absolute scale, $\chi^{\prime}$ at $100 \mathrm{kHz}$ increases at every oxidation step, even at the beginning of the oxidation, since the number of particles relaxing by Néel rotation $\left(\omega_{\text {char }} / 2 \pi>\right.$ $100 \mathrm{kHz}$ ) always increases. The first oxidation step already causes the break up of networks of particles into smaller units, so that the signal increases at $3 \mathrm{~Hz}$, but not many 
new Néel relaxing particles are created yet. The value at $100 \mathrm{kHz}$ stays approximately the same on an absolute scale but decreases when normalized to the $3 \mathrm{~Hz}$ value, which is the effect seen in Fig. 2C. Further oxidation does yield more Néel-relaxing particles, so that the $100 \mathrm{kHz}$ signal increases, even when compared to the $3 \mathrm{~Hz}$ value.

\subsection{Dynamic dipolar structures: a magnetite ferrofluid}

Complex magnetic susceptibility spectra of magnetite ferrofluid D are presented in Fig. 5. Assuming that surfactant-coated single magnetite particles $\left(a_{\mathrm{h}}=a_{\text {core }}+\right.$ $2 \mathrm{~nm}$ ) with a fixed magnetic moment exhibit Brownian relaxation at approximately $10 \mathrm{kHz}$ (see Eq. (3)), the observed characteristic frequency $\omega_{\text {char }} / 2 \pi$ of $100 \mathrm{~Hz}$ in the most concentrated sample (a) indicates the presence of large dipolar aggregates in which the magnetic moments are fixed $[5,16]$.

Upon dilution of the ferrofluid, the spectrum shifts to higher frequencies but still in the range where Brownian rotation occurs. This indicates the dissociation of dipolar structures into smaller units. Néel relaxation at frequencies above $100 \mathrm{kHz}$ becomes increasingly important. This suggests that the magnetic moments are not fixed in single uncorrelated particles, but that they are only arrested if a chain is formed. In small aggregates of two or three particles, the magnetic moments may possibly still react individually to the applied alternating magnetic field by Néel rotation.

As presented in Fig. 6a, cryo-TEM imaging of ferrofluid D corroborates the presence of dipolar structures. Dilution of the same ferrofluid by a factor 4.5 (Fig. 6b) yields shorter dipolar structures and more single particles, as expected for a chain-length distribution that results from a dynamic equilibrium. It is remarked that the flux-closure rings are relatively stable upon dilution and that they do not have a net contribution to the complex susceptibility spectrum, because of the way in which the dipoles are oriented head-to-tail in a circle.

\section{Conclusion}

Complex susceptibility measurements and cryo-TEM imaging were shown to give complementary characterizations of the dipolar structures, the first technique corresponding to a macroscopic average and the second giving microscopic information. The reversibility of dipolar chain formation in zero field was compared for two different systems, metallic iron ferrofluids and magnetite ferrofluids. With iron colloids, initially dynamic dipolar chains do not break up into single particles when they are gradually oxidized. Although the dipole moments of the individual particles decrease, the structures do not vanish but become chemically fixed during the oxidation process. This eventually leads to chains of iron-oxide core-shell units, of which the magnetic moments relax by the Néel mechanism. The oxidized iron systems are a clear example of irreversible aggregation. In contrast, in magnetite

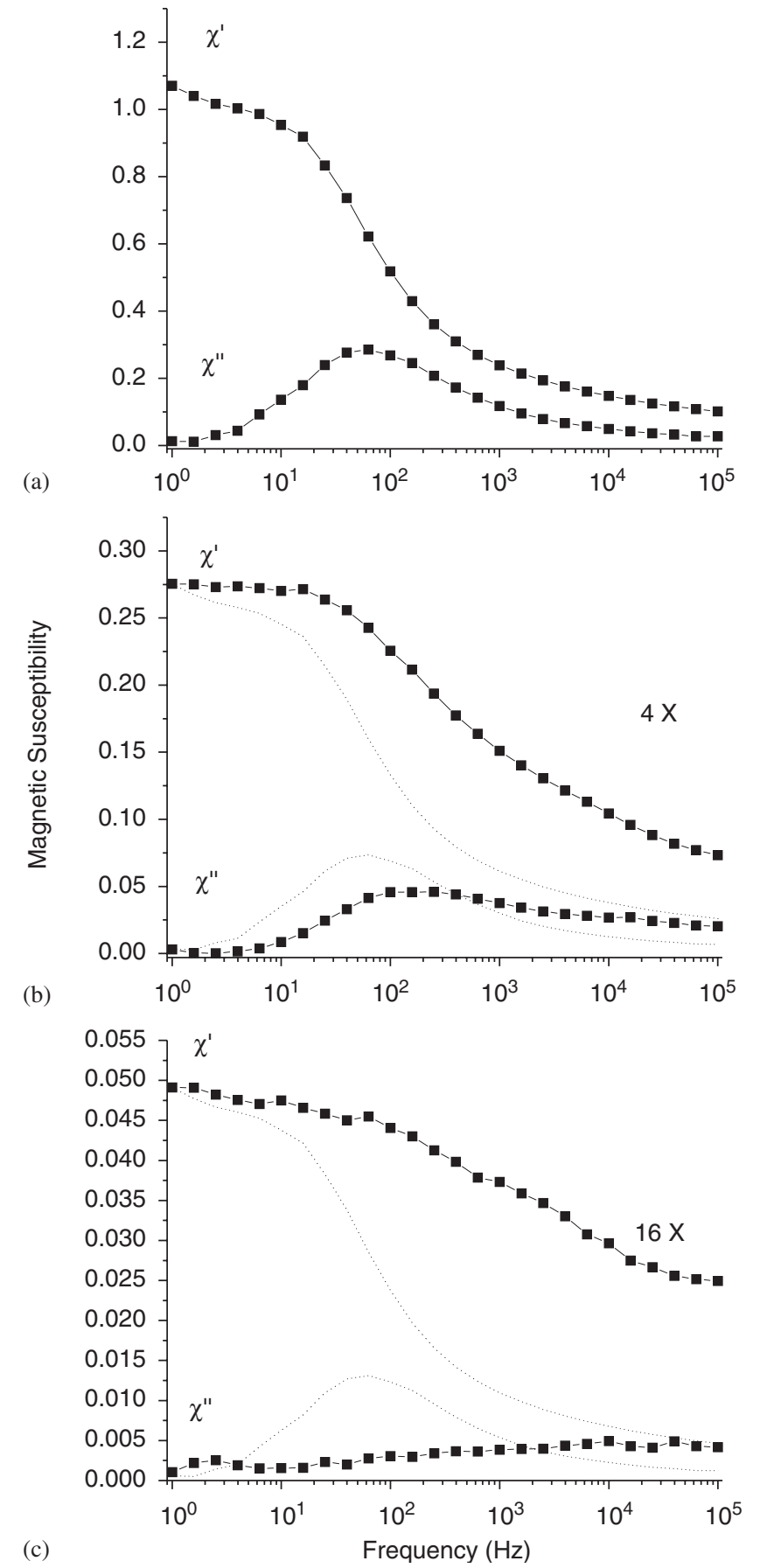

Fig. 5. Frequency dependence of the real $\chi^{\prime}$ and imaginary $\chi^{\prime \prime}$ components of the magnetic susceptibility of ferrofluid D. Spectrum (a) corresponds to a magnetite particle concentration of $332 \mathrm{mg} \mathrm{mL}^{-1},(\mathrm{~b}, \mathrm{c})$ are spectra of diluted samples of the same ferrofluid. The dilution factors relative to the original dispersion (situation (a)) are indicated in the figure $(4 \times$ and $16 \times)$. In $(b, c)$, a rescaled spectrum of the undiluted ferrofluid $D$ is included with dotted lines, for comparison.

dispersions, the dipolar chains do indeed break up upon dilution. A broad size distribution of magnetic chains remains, but the average chain length decreases upon dilution, leading to more single particles. This indicates that a dynamic equilibrium exists between chains of different sizes. 


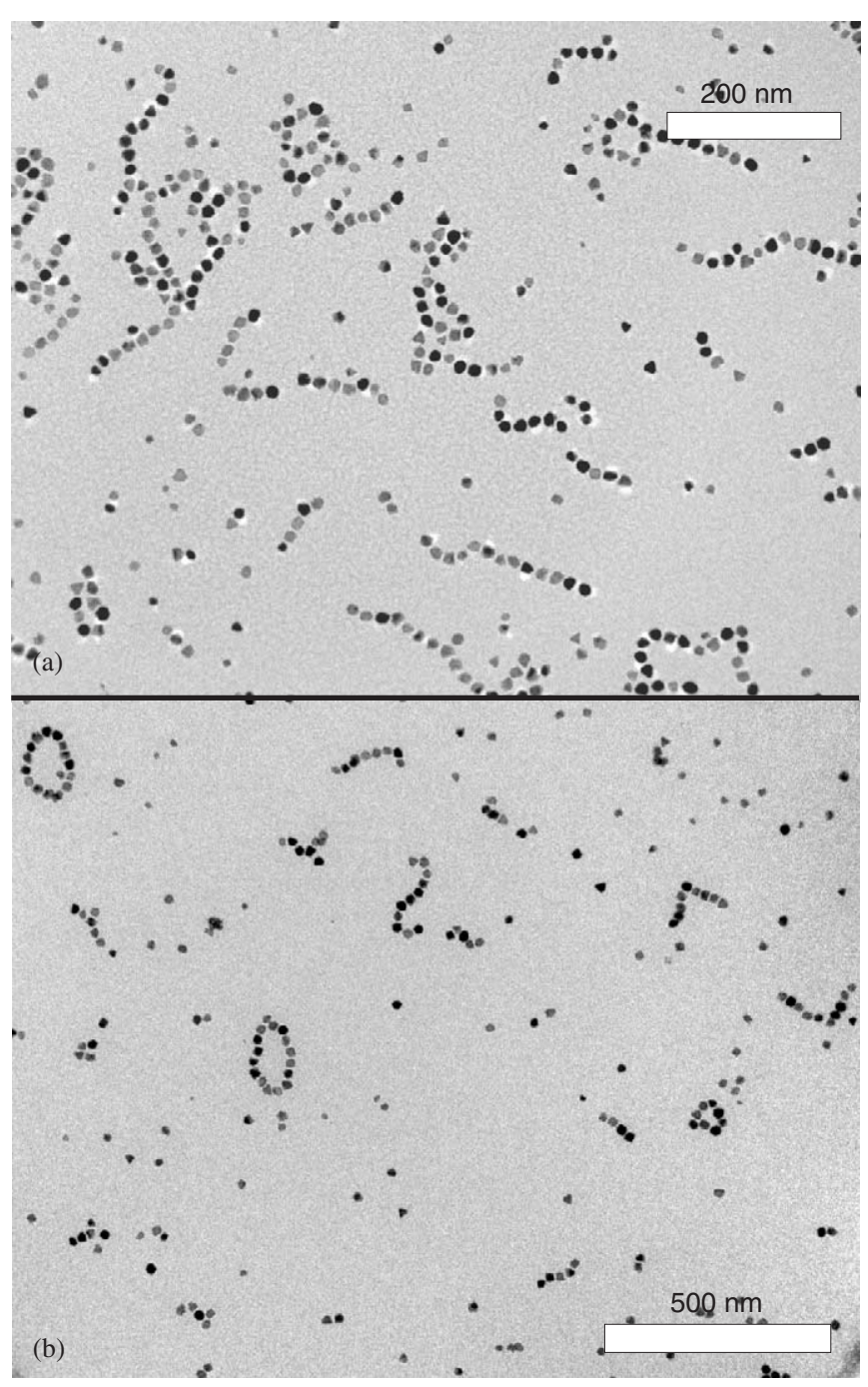

Fig. 6. Typical in situ cryo-TEM images of vitrified magnetite dispersion $\mathrm{D}$ (a) before and (b) after dilution of by a factor 4.5. The images indicate that the dipolar structures break up when the ferrofluid is diluted.

\section{Acknowledgments}

We are indebted to Dr. Stuart Charles, who suggested performing the present study. Dr. K. Butter is thanked for preparing and characterizing the iron dispersions, J. Meeldijk for helping with the cryo-TEM, and B. Kuipers for constructing the dynamic susceptibility setup.

\section{References}

[1] R.W. Chantrell, A. Bradbury, J. Popplewell, S.W. Charles, J. Phys. D 13 (1980) L119.

[2] J.M. Tavares, J.J. Weis, M.M.T. da Gama, Phys. Rev. E 65 (2002) 061201.

[3] K. Butter, P.H.H. Bomans, P.M. Frederik, G.J. Vroege, A.P. Philipse, Nature Mater. 2 (2003) 88.

[4] M. Klokkenburg, C. Vonk, E.M. Claesson, J.D. Meeldijk, B.H. Erné, A.P. Philipse, J. Am. Chem. Soc. (2004) 16706.

[5] B.H. Erné, K. Butter, B.W.M. Kuipers, G.J. Vroege, Langmuir 19 (2003) 8218.

[6] C.R. Brundle, T.J. Chuang, K. Wandelt, Surf. Sci. 68 (1977) 459.

[7] G.W.R. Leibbrandt, S. Deckers, M. Wiegel, F. Habraken, Surf. Sci. 244 (1991) L101-L106.

[8] D.H. Kim, S.S. Kim, H.H. Lee, H.W. Jang, J.W. Kim, M. Tang, K.S. Liang, S.K. Sinha, D.Y. Noh, J. Phys. Chem. B 108 (2004) 20213.

[9] K. Butter, A.P. Philipse, G.J. Vroege, J. Magn. Magn. Mater. 252 (2002) 1.

[10] K. Butter, K. Kassapidou, G.J. Vroege, A.P. Philipse, J. Colloid Interface Sci. 287 (2005) 485.

[11] K. Butter, P.H. Bomans, P.M. Frederik, G.J. Vroege, A.P. Philipse, J. Phys. Condens. Matter 15 (2003) S1451.

[12] C. Pathmamanoharan, N. Zuiverloon, A. Philipse, Progr. Colloid Polym. Sci. 115 (2000) 141.

[13] P.M. Frederik, P.H.H. Bomans, P.F.J. Laeven, F.J.T. Nijpels, Device for Preparing Specimens for a Cryo-electron Microscope, Netherlands Industrial Property Office (RO/NL) PCT/NL02/00189.

[14] P.C. Fannin, Adv. Chem. Phys. 104 (1998) 181.

[15] G. Xiao, S. Liou, A. Levy, S. Taylor, C. Chien, Phys. Rev. B 34 (1986) 7573.

[16] P.C. Fannin, A.T. Giannitsis, S.W. Charles, Eur. Phys. J. 12 (2000) 93. 